PROCEEDINGS OF THE AMERICAN MATHEMATICAL SOCIETY

Volume 124, Number 3, March 1996

\title{
EVERY ČECH-ANALYTIC BAIRE SEMITOPOLOGICAL GROUP IS A TOPOLOGICAL GROUP
}

\author{
AHMED BOUZIAD
}

(Communicated by Franklin D. Tall)

\begin{abstract}
Among other things, we prove the assertion given in the title. This solves a problem of Pfister.
\end{abstract}

\section{INTRODUCTION}

A semitopological (respectively, paratopological group) is a group endowed with a topology for which the product is separately (respectively, jointly) continuous. In $1957 \mathrm{R}$. Ellis [6] showed that every locally compact paratopological group is a topological group. This answered a question posed by A. D. Wallace in [19]. Moreover, in [20] W. Zelazko has shown that every completely metrizable paratopological group is a topological group. Later, in 1982, N. Brand [3] generalized both Ellis' and Zelazko's results by proving that every Čech-complete paratopological group is a topological group. A new and short proof of this result was given by H. Pfister [17] three years later. It had been well known for many years that every locally compact or completely metrizable semitopological group is a paratopological group ([7], [16] respectively). These results motivated Pfister in [17, Remarks] to ask whether every Cech-complete semitopological group is a paratopological group and, hence by Brand's result, a topological group.

In [2, Theorem 4.3] we show the following: Let $G$ be a Cech-complete semitopological group. Then $G$ is a topological group if and only if $G$ is paracompact. (The 'only if' part is a result by L. G. Brown [4].) Then, to answer Pfister's question it suffices to prove that every Čech-complete semitopological group is paracompact. The purpose of the present paper is to give a complete answer to Pfister's problem by a different method and in a general form. We prove that every Čech-analytic Baire semitopological group is a topological group (Theorem 3.3). The class of Cech-analytic spaces was introduced by D. H. Fremlin in an unpublished note of 1980 (cf. [12]). This class is sufficiently large to include all completely metrizable or locally compact spaces, and more generally, all Čech-complete spaces.

Theorem 3.3 is stated in Section 3 as a corollary of a somewhat more general statement (Theorem 3.2). Theorem 3.2 and Theorem 3.1 are settled in terms of $\mathrm{p}-\sigma$-fragmentability. In Section 2 , the concept of p- $\sigma$-fragmentability is introduced and some auxiliary results are established.

Received by the editors January 19, 1994.

1991 Mathematics Subject Classification. Primary 22A20, 54E18, 54H15, 57S25.

Key words and phrases. Semitopological group, topological group, generalized continuity, fragmentability. 


\section{Definitions AND PRELIMINARIES}

All topological spaces considered in this paper are supposed to be completely regular. Let $X$ be a topological space. We shall say that a subset $Y$ of $X$ is fragmented by a collection $\mathcal{U}$ of subsets of $X$ if each nonvoid subset of $Y$ has a nonvoid relatively open subset contained in some member of $\mathcal{U}$. The space $X$ is $\sigma$-fragmented by a cover $\mathcal{U}$ of $X$ (not necessarily related to the topology of $X$ ) if we can write $X=\bigcup_{n \in \mathbb{N}} X_{n}$ where, for each $n \in \mathbb{N}, X_{n}$ is fragmented by $\mathcal{U}$.

Recall that a sequence of covers $\left(\mathcal{U}_{n}\right)_{n \in \mathbb{N}}$ of $X$ is complete (cf. [12, p. 278]) if, whenever $\mathcal{F}$ is a filter base on $X$ such that each $\mathcal{U}_{n}$ has a member containing some member of $\mathcal{F}$, then $\bigcap\{\bar{F}: F \in \mathcal{F}\} \neq \varnothing$. We shall say that a sequence of covers $\left(\mathcal{U}_{n}\right)_{n \in \mathbb{N}}$ of $X$ is point-complete if, whenever $\mathcal{F}$ is a filter base on $X$ such that each $\mathcal{U}_{n}$ has a member $U_{n}$ containing some member of $\mathcal{F}$ such that $\bigcap_{n \in \mathbb{N}} U_{n} \neq \varnothing$, then $\bigcap\{\bar{F}: F \in \mathcal{F}\} \neq \varnothing$. It is clear that a complete sequence of covers of $X$ is a point-complete sequence of covers of $X$. We now introduce a concept which plays a fundamental role in this paper.

Definition 2.1. The space $X$ is called p- $\sigma$-fragmentable if $X$ has a point-complete sequence of covers $\left(\mathcal{U}_{n}\right)_{n \in \mathbb{N}}$ such that:

(1) $X$ is $\sigma$-fragmented by each $\mathcal{U}_{n}$,

(2) the collection $\left\{\bar{U}: U \in \mathcal{U}_{n+1}\right\}$ is a refinement of $\mathcal{U}_{n}$ for each $n \in \mathbb{N}$.

We shall say that a sequence of covers of $X$ satisfying Definition 2.1 is associated to the $\mathrm{p}-\sigma$-fragmentable space $X$.

Examples 2.2. (1) All metrizable and all Čech-complete spaces are p- $\sigma$-fragmentable. More generally all p-spaces are p- $\sigma$-fragmentable. Recall that a space $X$ is a p-space $([10])$ if $X$ has a sequence $\left(\mathcal{U}_{n}\right)_{n \in \mathbb{N}}$ of open covers such that if $x \in X$ and for each $n \in \mathbb{N}$ there is $U_{n} \in \mathcal{U}_{n}$ such that $x \in U_{n}$, then the set $K=\bigcap_{n \in \mathbb{N}} \bar{U}_{n}$ is compact and the sequence $\left(\bigcap_{i<n} \bar{U}_{i}\right)_{n \in \mathbb{N}}$ is an outer network for $K$. A family $\mathcal{N}$ of subsets of $X$ is an outer network for $K$ if for any open subset $U$ such that $K \subset U$ there exists $N \in \mathcal{N}$ such that $K \subset N \subset U$. The concept of a p-space was introduced by A. V. Arhangel'skii [1] in a different but equivalent form.

(2) Let $X$ be a space $\sigma$-fragmented by a lower semicontinuous metric (cf. [13]). If the metric topology is finer than the original one, then $X$ is p- $\sigma$-fragmented. One can use Lemma 2.4 below to show that not all spaces fragmented by a metric are $\mathrm{p}$ - $\sigma$-fragmented; this answers a question asked by the referee. This is the case of the Sorgenfrey line (see the paragraph after Lemma 2.4).

(3) Cech-analytic spaces are p- $\sigma$-fragmentable. (Recall that a space $X$ is Čechanalytic [12, Theorem 5.3] if $X$ is the projection on some compactification $X^{*}$ of $X$ of the intersection of a closed set and a $G_{\delta}$ subset of $X^{*} \times \mathbb{N}^{\mathbb{N}}$.) In fact, Theorem 5.7 of [12] says that a Čech-analytic space $X$ has a complete sequence of covers $\left(\mathcal{U}_{n}\right)_{n \in \mathbb{N}}$ satisfying (2) of 2.1 ; moreover, for each $n \in \mathbb{N}$ one can write $\mathcal{U}_{n}=\bigcup_{m \in \mathbb{N}} \mathcal{U}_{n, m}$, where for each $m \in \mathbb{N}, \mathcal{U}_{n, m}$ is an open cover of the subspace $X_{m}=\bigcup \mathcal{U}_{n, m}$ of $X$. Hence the space $X$ is $\sigma$-fragmented by each $\mathcal{U}_{n}$.

Following [15] a point $x \in X$ is called a q-point if it has a sequence of neighborhoods $\left(U_{n}\right)_{n \in \mathbb{N}}$ such that if $x_{n} \in U_{n}$, then the sequence $\left(x_{n}\right)_{n \in \mathbb{N}}$ has a cluster point in $X$. The space $X$ is called a q-space if every $x \in X$ is a q-point. We shall also need the following generalizations of continuity. Let $X$ and $Y$ be topological spaces, and let $f: X \rightarrow Y$. The mapping $f$ is called quasicontinuous at $x \in X$ [14] 
if for each neighborhood $V$ of $f(x)$ in $Y$ and for each neighborhood $U$ of $x$ in $X$ there is a nonvoid open set $O \subset U$ such that $f(O) \subset V$. The mapping $f$ is quasicontinuous if $f$ is quasicontinuous at every point $x \in X$. The mapping $f: X \rightarrow Y$ is subcontinuous at the point $x \in X$ [9] if for each net $\left(x_{\alpha}\right)$ in $X$ which converges to $x$, the net $\left(f\left(x_{\alpha}\right)\right)$ has a cluster point in $Y$.

The main result of this section is Theorem 2.7. To establish this result we need the following lemmas.

Lemma 2.3. Let $X$ be a Baire space, $Y$ a topological space and $f: X \rightarrow Y a$ quasicontinuous mapping. Suppose that $Y$ is $\sigma$-fragmented by the cover $\mathcal{U}$. Then the set $A$ of points $x \in X$ for which there exists $U \in \mathcal{U}$ and a neighborhood $V$ such that $f(V) \subset \bar{U}$ is a dense open subset of $X$.

Proof. It is clear that $A$ is open. Put $Y=\bigcup_{n \in \mathbb{N}} Y_{n}$ where each $Y_{n}$ is fragmented by $\mathcal{U}$. Let $\Omega$ be a nonvoid open subset of $X$ and let us show that there is a nonvoid open subset $O \subset \Omega$ of $X$ and $U \in \mathcal{U}$ such $f(O) \subset \bar{U}$. This will imply that $A$ is dense in $X$. Since $\Omega$ is a Baire space and since $\Omega \subset \bigcup_{n \in \mathbb{N}} f^{-1}\left(Y_{n}\right)$, there are a nonvoid open set $O_{1} \subset \Omega$ and $n \in \mathbb{N}$ such that $O_{1} \subset \overline{f^{-1}\left(Y_{n}\right)}$. In particular $f\left(O_{1}\right) \cap Y_{n} \neq \varnothing$. As $Y_{n}$ is fragmented by $\mathcal{U}$, there are an open set $W$ of $Y$ and $U \in \mathcal{U}$ such that $\varnothing \neq f\left(O_{1}\right) \cap Y_{n} \cap W \subset U$. Pick a nonvoid open subset $O$ of $X$ such that $O \subset O_{1}$ and $f(O) \subset W$; this is possible since $f$ is quasicontinuous. Let us show that $f(O) \subset \overline{f\left(O_{1}\right) \cap Y_{n} \cap W}$; this implies that $f(O) \subset \bar{U}$. Let $y \in f(O)$ and $V$ be a neighborhood of $y$ in $Y$; pick $x \in O$ such that $y=f(x)$. The mapping $f$ is quasicontinuous at $x$, hence there is a nonvoid open set $O_{2} \subset O$ of $X$ such that $f\left(O_{2}\right) \subset V \cap W$. It follows from $O_{2} \subset O \subset \overline{f^{-1}\left(Y_{n}\right)}$ that $O_{2} \cap f^{-1}\left(Y_{n}\right) \neq \varnothing$; hence

$$
\varnothing \neq f\left(O_{2}\right) \cap Y_{n} \cap f(O) \subset V \cap f\left(O_{1}\right) \cap Y_{n} \cap W .
$$

Lemma 2.4. Let $f: X \rightarrow Y$ be a quasicontinuous mapping where $X$ is a Baire space and $Y$ a p- $\sigma$-fragmentable space. Then the set of points of subcontinuity of $f$ is a dense subset of $X$.

Proof. Take a sequence of covers $\left(\mathcal{U}_{n}\right)_{n \in \mathbb{N}}$ associated to the p- $\sigma$-fragmentable space $Y$. For each $n \in \mathbb{N}$ let $A_{n}$ be the set of points $x \in X$ for which there is a neighborhood $V$ in $X$ and a member $U \in \mathcal{U}_{n}$ such that $f(V) \subset \bar{U}$. Let $A=\bigcap_{n \in \mathbb{N}} A_{n}$; by Lemma 2.3 the set $A$ is a dense $G_{\delta}$ subset of $X$. We show that $f$ is subcontinuous at every $x \in A$. Let $x \in A$ and $\left(x_{\alpha}\right)_{\alpha \in \Lambda}$ be a net in $X$ which converges to $x$. Let $\mathcal{F}=\left\{\left\{f\left(x_{\alpha}\right): \beta \leq \alpha\right\}: \beta \in \Lambda\right\}$; we must verify that $\bigcap\{\bar{F}: F \in \mathcal{F}\} \neq \varnothing$. For each $n \in \mathbb{N}$ pick $U_{n} \in \overline{\mathcal{U}}_{n+1}$ and $\beta_{n} \in \Lambda$ such that $f(x) \in \bar{U}_{n}$ and $\left\{f\left(x_{\alpha}\right): \beta_{n} \leq \alpha\right\} \subset \overline{U_{n}}$; since, for every $n \in \mathbb{N}$, the collection $\left\{\bar{U}: U \in \mathcal{U}_{n+1}\right\}$ is a refinement of $\mathcal{U}_{n}$, and since $\left(\mathcal{U}_{n}\right)_{n \in \mathbb{N}}$ is point-complete, we have $\bigcap\{\bar{F}: F \in \mathcal{F}\} \neq \varnothing$.

Denote by $L$ the Sorgenfrey line ([8]). The space $L$ is fragmented by the usual metric. The mapping $x \in \mathbb{R} \rightarrow x \in L$ is quasicontinuous but has no point of subcontinuity. Hence, by Lemma $2.4, L$ is not p- $\sigma$-fragmentable as is mentioned in Examples 2.2.2.

Remarks 2.5. Let $X$ be a Baire p- $\sigma$-fragmentable space and let $\left(\mathcal{U}_{n}\right)$ be a sequence of covers of $X$ associated to this space. For each $n \in \mathbb{N}$, let $A_{n}$ be the union of interiors of all elements in $\mathcal{U}_{n}$. By Lemma 2.3, the set $A=\bigcap_{n \in \mathbb{N}} A_{n}$ is a dense $G_{\delta}$ subset of $X$. 
(1) Every point $x \in A$ is a q-point of $X$. This follows from the point-completeness of the sequence $\left(\mathcal{U}_{n}\right)_{n \in \mathbb{N}}$.

(2) Suppose moreover that the sequence $\left(\mathcal{U}_{n}\right)_{n \in \mathbb{N}}$ is complete. Then the subspace $A$ of $X$ is Čech-complete. To show this fact, for each $n \in \mathbb{N}$ and for each $x \in A$ pick by Lemma 2.3 a neighborhood $V_{n}^{x}$ of $x$ in $X$ and $U_{n}^{x} \in \mathcal{U}_{n}$ such that $\overline{V_{n}^{x}} \subset$ $U_{n}^{x} \subset A_{n}$; then the sequence of open covers $\left(\mathcal{V}_{n}\right)_{n \in \mathbb{N}}$ of the space $A$, defined by $\mathcal{V}_{n}=\left\{V_{n}^{x} \cap A: x \in A\right\}$, is complete.

In this paper we investigate continuity of separately continuous group operations. The following lemma implies in particular that every separately continuous mapping $f: X \times Y \rightarrow Z$, where $X$ is a Baire p-space and $Y$ a q-space, is quasicontinuous. Other results of the same type have been obtained in the past (see [11] and the bibliography in this paper). To establish this lemma we use the following topological game.

Christensen's game (cf. [5]). Let $X$ be a topological space. The game $\mathcal{G}_{\sigma}$ is a two-player game. An instance of $\mathcal{G}_{\sigma}$ is a sequence of triplets $\left(\left(U_{n}, V_{n}, x_{n}\right)\right)_{n \in \mathbb{N}}$ defined inductively as follows: Player $\beta$ begins and chooses a nonempty open set $U_{0}$ of $X$; player $\alpha$ then chooses a nonempty open set $V_{0} \subset U_{0}$ and a point $x_{0} \in X$. When $\left(U_{i}, V_{i}, x_{i}\right), 0 \leq i \leq n-1$, have been defined, player $\beta$ picks a nonempty open set $U_{n} \subset V_{n-1}$ and player $\alpha$ chooses a nonempty open set $V_{n} \subset U_{n}$ and a point $x_{n} \in X$. Player $\alpha$ wins if

$$
\left(\bigcap U_{n}\right) \cap \overline{\left\{x_{n}: n \in \mathbb{N}\right\}} \neq \varnothing .
$$

In $[2$, Proposition 3.6] we demonstrate that every Baire p-space $X$ is $\sigma$ - $\beta$-defavorable, which means player $\beta$ has no winning strategy in the game $\mathcal{G}_{\sigma}$ on $X$. By Lemma 2.3 (see also 2.5) the same proof of [2, Proposition 3.6] allows more generally that every p- $\sigma$-fragmentable Baire space is $\sigma$ - $\beta$-defavorable. We shall use this fact in this paper.

Lemma 2.6. Let $X$ be a $\sigma$ - $\beta$-defavorable space, $Y$ a space with a dense subset of $q$-points, $Z$ a topological space and $f: X \times Y \rightarrow Z$ a separately continuous mapping. Then $f$ is quasicontinuous.

Proof. Let us suppose the opposite. Choose a point $(a, b) \in X \times Y$ such that $f$ is not quasicontinuous at $(a, b)$. Let $W$ be an open set of $Z$ which contains $f(a, b)$ and let $U \times V$ be an open paving of $X \times Y$ which contains $(a, b)$, such that for each nonvoid open set $O \subset U \times V$ one has $f(O) \not \subset W$. Without loss of generality we may assume that $b$ is a q-point of $Y$. Let $\varphi: Z \rightarrow \mathbb{R}$ be a continuous mapping such that $\varphi(f(a, b))=1$ and $\varphi\left(W^{c}\right) \subset\{0\}$ (recall that $Z$ is a completely regular space). Let $\psi$ denote the separately continuous map $f \circ \varphi: X \times Y \rightarrow \mathbb{R}$. Pick a sequence $\left(O_{n}\right)_{n \in \mathbb{N}}$ of neighborhoods associated to the q-point $b \in Y$. We shall define a strategy $\tau$ for player $\beta$ in the game $\mathcal{G}_{\sigma}$ on the space $X$ as follows: To begin $\beta$ plays the nonempty open subset $\tau(\varnothing)=U \cap\{x \in X: \psi(x, b)>0\}$ of $X$ and chooses a point $\left(x_{0}, y_{0}\right) \in \tau(\varnothing) \times\left(O_{0} \cap V\right)$ such that $\psi\left(x_{0}, y_{0}\right)=0$. At the $(n+1)$ th stroke, if player $\alpha$ has played $\left(\left(V_{0}, a_{0}\right), \ldots,\left(V_{n}, a_{n}\right)\right)$, then $\beta$ chooses $x_{n+1} \in V_{n}$ and $y_{n+1} \in O_{n+1} \cap V$, satisfying the conditions

$$
\begin{gathered}
\left|\psi\left(a_{i}, b\right)-\psi\left(a_{i}, y_{n+1}\right)\right| \leq 1 /(n+1) \quad \text { for each } i=0, \ldots, n, \\
\psi\left(x_{n+1}, y_{n+1}\right)=0,
\end{gathered}
$$


and $\beta$ plays the nonempty open set

$$
\tau\left(\left(V_{0}, a_{0}\right), \ldots,\left(V_{n}, a_{n}\right)\right)=\left\{x \in V_{n}\left|\psi\left(x, y_{n+1}\right)\right|<1 /(n+1)\right\} .
$$

Since the space $X$ is $\sigma$ - $\beta$-defavorable, there is for $\alpha$ a winning game $\left(V_{n}, a_{n}\right)_{n \in \mathbb{N}}$ against the strategy $\tau$. Let $x \in\left(\bigcap_{n \in \mathbb{N}} V_{n}\right) \cap \overline{\left\{a_{n}: n \in \mathbb{N}\right\}}$ and let $y \in Y$ be some cluster point of the sequence $\left(y_{n}\right)_{n \in \mathbb{N}}$ in $Y$. Then we easily obtain the contradiction $0=\psi(x, y)=\psi(x, b)=1$.

The key for the proof of our main result (Theorem 3.1) is

Theorem 2.7. Let $X$ be a $\sigma$ - $\beta$-defavorable space, $Y$ a space with a dense set of $q$-points and $Z$ a p- $\sigma$-fragmentable space. Suppose that the product $X \times Y$ is a Baire space. Then every separately continuous mapping $f: X \times Y \rightarrow Z$ is subcontinuous at each point of a dense $G_{\delta}$ subset of $X \times Y$.

Proof. This follows from Lemma 2.6 and Lemma 2.4 .

\section{Applications to semitopological groups}

Let $G$ be a group endowed with a topology. Let us recall that the group $G$ is said to be semitopological if for every $g \in G$ the mappings $h \in G \rightarrow h g \in G$ and $h \in G \rightarrow g h \in G$ are continuous. It is called paratopological if the mapping $(g, y) \in G \times G \rightarrow g h \in G$ is continuous.

Theorem 3.1. Let $G$ be a semitopological group and suppose that the product $G \times G$ is a Baire space. If $G$ is $p$ - $\sigma$-fragmentable, then $G$ is a paratopological group.

Proof. We must show that the product mapping $(g, h) \in G \times G \rightarrow g h \in G$ is continuous. It suffices to prove that for every net $\left(\left(g_{\alpha}, h_{\alpha}\right)\right)_{\alpha \in \Lambda}$ in $G \times G$ which converges to the point $(g, h)$, the point $g h$ is a cluster point of the net $\left(g_{\alpha} h_{\alpha}\right)_{\alpha \in \Lambda}$ in $G$. Let $\left(g_{\alpha}, h_{\alpha}\right)_{\alpha \in \Lambda}$ be such a net. Consider by Remarks 2.5 and Theorem 2.7 a point $(a, b) \in G \times G$ of subcontinuity of the product mapping in $G$. We have $\lim a g^{-1} g_{\alpha}=a$ and $\lim h_{\alpha} h^{-1} b=b$, hence the net $\left(a g^{-1} g_{\alpha} h_{\alpha} h^{-1} b\right)_{\alpha \in \Lambda}$ has a cluster point in $G$; as the multiplication in $G$ is separately continuous the net $\left(g_{\alpha} h_{\alpha}\right)_{\alpha \in \Lambda}$ must have a cluster point $y \in G$. To end the proof we show that $y=g h$. Since $G$ is completely regular, it is sufficient to show that $f(g h)=f(y)$ for any continuous real function on $G$. Let $f: G \rightarrow \mathbb{R}$ be such a function. Let $B$ denote the set of q-points of $G ; B$ is a dense subset of $G$ by Remarks 2.5. (Since $G$ is homogeneous, we have $B=G$; but we do not use this fact.) Pick $c \in B$. The mapping $\varphi:(u, v) \in G \times G \rightarrow f(u v) \in \mathbb{R}$ is separately continuous, hence by $[2$, Theorem 2.3] there is a dense subset $A$ of $G$ such that $\varphi$ is continuous at every point of $A \times\{c\}$. Let $u \in A$. We have $\lim u g^{-1} g_{\alpha}=u$ and $\lim h_{\alpha} h^{-1} c=c$, hence $\lim f\left(u g^{-1} g_{\alpha} h_{\alpha} c\right)=f(u c)$. Then $f\left(u g^{-1} y h^{-1} c\right)=f(u c)$ for each $u \in A$. Since $A$ is a dense subset of $G$, it follows that $f\left(y h^{-1} c\right)=f(g c)$; and since $B$ is also dense in $G$, we obtain $f(y)=f(g h)$. This completes the proof.

Let $G$ be a p- $\sigma$-fragmentable semitopological group. Suppose that $G$ is p- $\sigma$ fragmented by a complete sequence of covers. If $G$ is a Baire space, then by Remarks $2.5 G$ has a dense Cech-complete subspace. It follows that $G \times G$ is a Baire space, and then by Theorem $3.1 G$ is a paratopological group. Now, by [2, Theorem $4.2] G$ is a topological group. This proves the following result.

Theorem 3.2. Let $G$ be a semitopological Baire group. If $G$ is $p$ - $\sigma$-fragmentable by a complete sequence of covers, then $G$ is a topological group. 
Since every Čech-analytic space is p- $\sigma$-fragmentable by a complete sequence of covers (cf. 2.2(3)), the following is a corollary of 3.2 .

Theorem 3.3. Every Čech-analytic Baire semitopological group is a topological group.

The next particular case of 3.3 answers affirmatively Pfister's problem mentioned in the introduction.

Corollary 3.4. Every Čech-complete semitopological group is a topological group.

Remark 3.5. Brand proves in [3] that every locally Čech-complete paratopological group is a topological group. Hence, as asked by the referee, is it natural to try to prove that every locally Cech-complete semitopological group is paratopological and hence a topological group? In a private conversation J. P. Troallic solves this question as follows: Let $G$ be a locally Čech-complete semitopological group and note that $G$ is a q-space and a $\sigma$ - $\beta$-defavorable space. Let $W$ be a nonvoid open Čech-complete subspace of $G$. By Lemma 2.6 the group multiplication $\pi:(g, h) \in$ $G \times G \rightarrow g h \in G$ is quasicontinuous, hence there exists a nonvoid open paving $U \times V$ of $G \times G$ such that $\pi(U \times V) \subset W$. Then, by Theorem 2.7, the mapping $\pi: U \times V \rightarrow W$ has at least a point of subcontinuity. Now, by the proof of Theorem 3.1, it follows that the group multiplication is continuous.

Note. In his (her) comments on a second version of this paper, the referee pointed out to us that E. A. Reznichenko has announced without proof in [18] our Corollary 3.4 .

\section{REFERENCES}

[1] A. V. Arhangel'skii, On a class containing all metric and locally bicompact spaces, Dokl. Akad. Nauk. SSSR 151 (1963), 751-754; Eng. Trans., Sov. Math. Dokl. 4 (1963), 1051-1055. MR 27:2959

[2] A. Bouziad, The Ellis theorem and continuity in groups, Topol. Appl. 50 (1993), 73-80. MR 94i: 22002

[3] N. Brand, Another note on the continuity of the inverse, Arch. Math. 39 (1982), 241-245. MR 84b:22001

[4] L. G. Brown, Topological complete groups, Proc. Amer. Math. Soc. 35 (1972), 593-600. MR 46:7435

[5] J. P. R. Christensen, Joint continuity of separately continuous functions, Proc. Amer. Math. Soc. 82 (1981), 455-461. MR 82h:54012

[6] R. Ellis, A note on the continuity of the inverse, Proc. Amer. Math. Soc. 8 (1957), 372-373. MR 18:745d

[7] R. Ellis, Locally compact transformation groups, Duke Math. J. 24 (1957), 119-125. MR 19:561b

[8] R. Engelking, General Topology, Heldermann Verlag, Berlin (1989). MR 91c:54001

[9] R. V. Fuller, Relations among continuous and various non continuous functions, Pac. J. Math. 25 (1968), 495-509. MR 37:3536

[10] G. Gruenhage, Generalized metric spaces, In K. Kunen and J. E. Vaughan (eds), Handbook of Set-Theoretic Topology (Elsevier Science Publishers) (1984), 423-501. MR 86h:54038

[11] G. Hansel and J. P. Troallic, Quasicontinuity and Namioka's Theorem, Topol. Appl. 46 (1992), 135-149. MR 94a:54047

[12] R. W. Hansell, Descriptive Topology, In M. Hušek and J. van Mill (edts), Recent Progress in General Topology (Elsevier Science Publishers) (1992), 275-315. CMP 93:15

[13] J. E. Jayne and C. A. Rogers, Borel selectors for upper semicontinuous set-valued maps, Acta Math. 155 (1985), 41-79. MR 87a:28011

[14] S. Kempisty, Sur les fonctions quasicontinues, Fund. Math. 19 (1932), 184-197. 
[15] E. Michael, A note on closed maps and compact sets, Israel J. Math. 2 (1964), 173-176. MR 31:1659

[16] D. Montgomery, Continuity in topological groups, Bull. Amer. Math. Soc. 42 (1936), 879-882.

[17] H. Pfister, Continuity of the inverse, Proc. Amer. Math. Soc. 95 (1985), 312-314. MR 87a:22004

[18] E. A. Reznichenko, Continuity in complete groups, Abstract in Tenth Summer Conference on General Topology and Applications, Amsterdam 1994, p. 135.

[19] A. D. Wallace, The structure of topological semigroups, Bull. Amer. Math. Soc. 61 (1955), 95-112. MR 16:796d

[20] W. Zelazko, A theorem on $B_{0}$ division algebras, Bull. Acad. Pol. Sci. 8 (1960), 373-375. MR 23:A3198

Analyse et Modèles Stochastiques, URA C.N.R.S. 1378, U.F.R. Des Sciences de Rouen, 76821 Mont Saint Aignan cedex, France

E-mail address: ahmed.bouziad@univ-rouen.fr 